\title{
Preoperative Ultrasound to Map the Three- dimensional Anatomical Distribution of the Lateral Femoral Cutaneous Nerve in Direct Anterior Approach for Total Hip Arthroplasty
}

\section{Yu Zhang}

Nanjing Drum Tower Hospital: Nanjing University Medical School Affiliated Nanjing Drum Tower Hospital

Yao Yao

Nanjing Drum Tower Hospital: Nanjing University Medical School Affiliated Nanjing Drum Tower Hospital

\section{Yexian Wang}

Nanjing Drum Tower Hospital: Nanjing University Medical School Affiliated Nanjing Drum Tower Hospital

\section{Zaikai Zhuang}

Nanjing Drum Tower Hospital: Nanjing University Medical School Affiliated Nanjing Drum Tower Hospital

\section{Ying Shen}

Nanjing Drum Tower Hospital: Nanjing University Medical School Affiliated Nanjing Drum Tower Hospital

Qing Jiang ( $\nabla$ qingj@nju.edu.cn )

Drum Tower Hospital,School of Medicine, Nanjing University https://orcid.org/0000-0002-2552-9686

\section{Dongyang Chen}

Nanjing Drum Tower Hospital: Nanjing University Medical School Affiliated Nanjing Drum Tower Hospital

\section{Research Article}

Keywords: ultrasound, lateral femoral cutaneous nerve, direct anterior approach, total hip arthroplasty

Posted Date: September 8th, 2021

DOl: https://doi.org/10.21203/rs.3.rs-142027/v2

License: (c) (i) This work is licensed under a Creative Commons Attribution 4.0 International License.

Read Full License 
Page 2/16 


\section{Abstract}

Background政he postoperative complaints of hypoaesthesia or a burning sensation due to lateral femoral cutaneous nerve (LFCN) injury in patients is not yet solved. The present study aimed to identify the three-dimensional (3D) distribution of LFCN using preoperative ultrasound and evaluate the rate of injury in direct anterior approach for total hip arthroplasty.

Methods $\rrbracket$ A total of 59 patients ( 28 males and 31 females, age $69.0 \pm 4.6$ years $\varangle \mathrm{BMI} 24.7 \pm 3.0 \mathrm{~kg} / \mathrm{m}^{2}$ ) were randomly allocated to the ultrasound group and 58 patients ( 28 males and 30 females, age $68.5 \pm$ 4.5 years, BMI $24.8 \pm 2.8 \mathrm{~kg} / \mathrm{m}^{2}$ ) were in the control group. Surgeons received the data of $3 \mathrm{D}$ distribution of LFCN only in the ultrasound group before surgery with respect to the direction, the depth on the skin, and the length to tensor fasciae latae (TFL). The anatomical characteristics of LFCN in the surgical region were summarized. At 1 and 3 months post-surgery, the rate of LFCN injury and abnormal sensitive area were evaluated in both groups.

Results $\triangle$ There was a significant consistency in gender, age and BMl of these two groups ( $P>0.05)$. Based on the data from the ultrasound group, over $90 \%$ of patients had one or two branches of LFCN. LFCN always courses in the fascia layer, the depth ranged from $6.8 \pm 2.6(3.0 \sim 12.0) \mathrm{mm}$ to $11.1 \pm 3.4(4.0 \sim 17.0)$ $\mathrm{mm}$ and depended on the thickness of the subcutaneous fat, and length was $3.3 \pm 4.6(-5.0 \sim 10.0) \mathrm{mm}$ at proximal part and $-2.7 \pm 4.7(-10.0 \sim 8.0)$ at distal end to the medial edge of TFL. Both the rate of LFCN injury and abnormal sensory area in the ultrasound group were significantly lower than those in the control group (3.4\% VS $25.9 \%, P=0.001$, at 1 month; $3.4 \%$ VS $22.4 \%, P=0.005$, at 3 months).

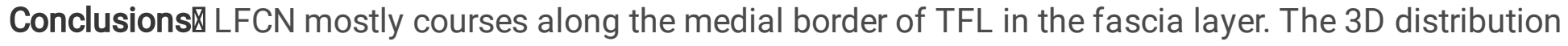
of LFCN using preoperative ultrasound mapping could help the surgeons to evaluate the risk of injury preoperatively and decrease the rate of injury during the operation. However, some branch injuries, especially for the fan type LFCN, could not be avoided.

\section{Background}

The direct anterior approach (DAA) is increasingly preferred by surgeons when patients accept hemiarthroplasty (HTA) or total hip arthroplasty (THA) [1,2]. This preference could be attributed to the reasons that DAA is a minimal soft invasion approach and uncovers the joint capsule through intermuscular and inter-nervous plane[3, 4]. Compared to other approaches, such as direct lateral, anterolateral and posterior approach, patients after DAA THA had improved early ambulation capacity, fewer reoperations, enhanced functional recovery and a low dislocation rate [5-9].

However, a patient's anxiety is due to lateral femoral cutaneous nerve (LFCN) injury that results in hypesthesia, dysesthesia or pain in the anterolateral aspect of the thigh [10-12]. This is the main complaint of the patients after DAA and causes a low satisfaction rate despite the high score of the hip function. Some studies from the clinic or cadaveric hips reported that the rate of LFCN injury was $3.29 \%-81.00 \%[10,11,13,14]$. This huge gap between different studies may be partially caused by 
surgical technique, including nerve stretching, compression, laceration, and suturing; however, the high anatomical variant rate of LFCN distribution and femoral offset, was at a higher risk for surgical injury [15-17]. The LFCN, derived from the lumbar nerve 2-3, crosses the iliacus obliquely, and then runs toward the anterior superior iliac spine (ASIS). After passing ASIS and piercing inguinal ligament, the 2 to 4 branches innervate the anterolateral aspect of the respective thigh. About $62 \%$ of the branches entered the proximal aspect of the thigh medial to the ASIS, and 38\% entered just above or lateral to the ASIS[17]. In addition, the fan-type branching pattern has been reported and cannot be avoided in DAA approach to the hip joint, and the LFCN injury rate of $90 \%$ in the fan-type group was significantly higher than $28.6 \%$ of in the non-fan-type group[18]. Therefore, finding a simple and efficient method to help surgeons avoid injuring LFCN, especially in patients with distribution variation, is critical.

Many studies have reported that damage to LFCN can be avoided by the preoperative identification of its distribution using ultrasound, which has higher sensitivity and equal specificity to magnetic response imaging (MRI) in noninvasive peripheral nerve visualization[16, 19-21]. Preoperative ultrasound maps the distribution of LFCN in the skin and the distal incision. However, the position of LFCN marking on the skin is easily changed with the skin in different positions of the hip joint, especially in the elderly with loose and wrinkled skin. Thus, it is not a good decision to only use skin mapping of LFCN as the reference. Herein, the three-dimensional (3D) anatomical distribution of LFCN, using ultrasound is employed to locate its position relative to the skin and muscles. We hypothesized that preoperative ultrasound identified the 3D anatomical distribution of LFCN, which decreases the rate of injury, and summarizes the distribution to help the surgeons avoid LFCN during the operation.

\section{Methods}

A total of 120 patients were randomly assigned to the control and ultrasound groups, and underwent DAA THA from September 2019 to June 2020. All patients provided informed consent, and the protocol was approved by our Faculty of Medicine-Institutional Ethics Review Board (ref. IRB 2021-413-01). Inclusion criteria: patients suffered from a fracture of neck of femur, femoral head necrosis, or osteoarthritis of the hip joint and need primary hip arthroplasty. Exclusion criteria: patients had a poor cardiopulmonary function, and hence, could not be burdened with the crisis of anesthesia and surgery and experienced other operations in the same hip joint with scar or anatomical disorder; the patients with a history of neurological abnormalities in the thigh were excluded. Moreover, general characteristics, including age, sex, and body mass index (BMI), were evaluated.

\section{Ultrasound mapping}

In the ultrasound group, two skilled physiatrists with neuromuscular ultrasound experience examined the 3D distribution using an ultrasound machine (SonoSite M-turbo, USA) with a $10 \mathrm{MHz}$ linear array transducer to detect the stem of LFCN at beginning. Then, the superior small branches would be detected via changing the frequencies probe based on the depth of LFCN stem. The ASIS and sartorius muscle 
was used as a reference to describe the continuous course of LFCN. Three cross-sectional areas were selected to trace the position of LFCN relative to skin and tensor fasciae latae (TFL). The first point was that LFCN left the pelvis near ASIS and inguinal ligament (IL), and the second and third recording points were $5 \mathrm{~cm}$ and $10 \mathrm{~cm}$ distal to the ASIS, respectively. The depths of LFCN to the skin at the three points were recorded as D1, D 2, and D3, respectively. The lengths of LFCN to the medial edge of TFL at the three points were recorded as $L 1, L 2$, and $L 3$, respectively. If $L F C N$ was located on the medial side of $T F L$, it was recorded as $-L$, and if lateral, it was $+L$ (figure 1. a, b). Finally, the course of the LFCN was mapped on the skin using a marker pen. These data will be taken as reference for surgeons to avoid damaging LFCN during operation (figure 1. c, d). In the control group, nothing tracked the course of LFCN.

\section{Surgical technique}

All surgeries were performed by one experienced DAA surgeon and three assistant surgeons. After intravenous anesthesia, the participant lied on a standard surgical bed in the supine position, and the hip joint that required arthroplasty was $10 \mathrm{~cm}$ higher than the other side. The skin incision was $2 \mathrm{~cm}$ lateral from ASIS and proceeded distally for about $8-10 \mathrm{~cm}$ that was parallel to the line of ASIS and lateral of the patella. Briefly, after subcutaneous and fascia dissection, the sartorius, temoriss, and tensor fascia were isolated, the muscle interval was uncovered, and the branches of lateral femoral circumflex artery ligated. The anterior capsulotomy and femoral neck osteotomy were performed, and then the acetabulum was exposed. The acetabulum was prepared using different sizes of offset reamers at $40-45^{\circ}$ abduction and $15^{\circ}$ anteversion until the surface oozed blood. After the installation of an artificial prosthesis of the acetabulum, the femoral preparation continued in a modified figure-four position of the leg with $45^{\circ}$ hyperextension and elevation of the femur by a double-tipped retractor behind the greater trochanter. The distance from the horizontal line of the great trochanter to the center of the rotation of the femoral head based on the result of pelvic radiograph was used to evaluate the length of the leg, and the range of movement was effectuated to assess the stability of leg. After introducing the artificial femoral component and head, the deep fascia, subcutaneous tissue, and intracutaneous were sutured step by step.

To identify the consistent results between preoperative ultrasound data and anatomical positions, five patients were informed and consented to expose the LFCN in surgery. Based on the preoperative ultrasound 3D distribution data, including the depth to skin, the mapping path and the length to TFL, we accomplished to show the anatomy of the LFCN intraoperation. It demonstrated the nerve located medical side of our incision and provided practical parameters for surgeons to keep the suture at an appropriate distance from border of incision while avoiding the LFCN. (Figure 2)

\section{Evaluation of LFCN injury in patients}

The operation time was calculated and compared between ultrasound group and control group because that the longer time was used to pull and expose surgical field and the more possible to injury nerve. At 1 
month and 3 months after surgery, all patients underwent follow-up in the Outpatient Department. Also, the abnormal sensation in the anterolateral thigh, including hypesthesia, dysesthesia, numbness, and pain was assessed. If a patient experiences abnormal sensation, he/she would be asked to mark the region area using a black marking pen (figure 3). This area was estimated by multiplying the longest diameter with the shortest diameter. An experienced doctor assessed the function of hip joint after DAA THA surgery.

\section{Statistics}

As the continuous variables are presented as mean $\pm S D$, Student's t-test was used to analyze data for normal distribution and Mann-Whitney U-test for abnormal distribution. On categorical variables, Fisher's exact test was used. $P<0.05$ indicated the statistical significance. It was calculated that 45 patients were required in each group to detect a difference in the rate of LFCN injury with a standard deviation of 14.8, $90 \%$ power and a two-sided alpha error of 0.05. Statistical Package for the Social Science version 23.0 (INM Corporation, Armonk, NY, USA) was utilized to analyze these data.

\section{Results}

\section{Demographics, Baseline patient information, and the 3D distribution of LFCN}

A total of 117 patients completed the follow-up at 1 month and 3 months after operation. The ultrasound group had 59 patients ( 28 men and 31 women; average age, $69.0 \pm 4.6$ years ( $58 \sim 79$ years)), while the control group had 58 patients ( 28 men and 30 women; average age, $68.5 \pm 4.5$ years ( $60 \sim 79$ years)). Of these, $28(23.9 \%)$ patients suffered from femoral neck fracture (FNF), 54 (46.2\%) from femoral head necrosis (FHN), and $35(29.9 \%)$ had osteoarthritis (OA). The mean BMI was $24.7 \pm 3.0(17.2 \sim 31.5) \mathrm{kg} / \mathrm{m}^{2}$ and $24.8 \pm 2.8$ (18.5 31.2) in the ultrasound and control groups, respectively (Table 1). In the ultrasound group, 30 (54.5\%) of LFCN were identified one branch, 23 (41.8\%) had two branches, and 2 (3.7\%) had at least three branches in the surgical region. The distance from the skin surface to LFCN was termed as D1, D2, and D3. The results of D1, D2, and D3 were $6.8 \pm 2.6(3.0 \sim 12.0) \mathrm{mm}, 9.2 \pm 2.8(3.0 \sim 15.0) \mathrm{mm}$, and $11.1 \pm 3.4(4.0 \sim 16.0) \mathrm{mm}$, respectively. The LFCN courses in the fascia that is on the surface of the sartorius and TLF within $10 \mathrm{~cm}$ from the far end of ASIS. The length from LFCN to the medial edge of TLF named L1, L2, and L3 was $3.3 \pm 4.6(-5.0 \sim 10.0) \mathrm{mm}, 0 \pm 4.1(-10.0 \sim 7.0) \mathrm{mm}$, and $-2.7 \pm 4.7(-10.0$ 8.0) $\mathrm{mm}$, respectively. (Table 2) 
Table 1

Demographic and medical history in patients with and without ultrasound definition of LFCN.

\begin{tabular}{|c|c|c|c|c|c|}
\hline & & Ultrasound group & Control group & $P$ & $t / x^{2}$ \\
\hline \multirow[t]{2}{*}{ Sex } & Male & $28(47.5 \%)$ & $28(48.3 \%)$ & \multirow[t]{2}{*}{1.000} & \multirow{2}{*}{$\chi^{2}=0.008$} \\
\hline & Female & $31(52.5 \%)$ & $30(51.7 \%)$ & & \\
\hline \multicolumn{2}{|c|}{ Age (years) } & $69.0 \pm 4.6(58 \sim 79)$ & $68.5 \pm 4.5(60 \sim 79)$ & 0.608 & $t=0.514$ \\
\hline \multicolumn{2}{|l|}{ BMI } & $24.7 \pm 3.0(17.2 \sim 31.5)$ & $24.8 \pm 2.8(18.5 \sim 31.2)$ & 0.827 & $t=0.219$ \\
\hline \multirow[t]{3}{*}{ Disease } & FNF & $15(25.4 \%)$ & $13(22.4 \%)$ & \multirow[t]{3}{*}{0.163} & \multirow[t]{3}{*}{$\chi^{2}=0.922$} \\
\hline & $\mathrm{FHN}$ & $27(45.8 \%)$ & $27(46.6 \%)$ & & \\
\hline & OA & $17(28.8 \%)$ & $18(31.0 \%)$ & & \\
\hline
\end{tabular}

Table 2

$D$ from skin and $L$ from the medical edge of TFL.

\begin{tabular}{|lll|}
\hline \multicolumn{1}{|c|}{$\mathbf{D}(\mathrm{mm})$} & $\mathrm{L}(\mathrm{mm})$ \\
\hline 1 & $6.8 \pm 2.6(3.0 \sim 12.0)$ & $3.3 \pm 4.6(-5.0 \sim 10.0)$ \\
\hline 2 & $9.2 \pm 2.8(3.0 \sim 15.0)$ & $0 \pm 4.1(-10.0 \sim 7.0 .0)$ \\
\hline 3 & $11.1 \pm 3.4(4.0 \sim 17.0)$ & $-2.7 \pm 4.7(-10.0 \sim 8.0)$ \\
\hline D, Depth; L, Length. & \\
\hline
\end{tabular}

\section{The post-surgery function of the hip joint and the injury of LFCN}

Before surgery, the preoperative hip Harris scores were assessed in these two groups, and patients with femoral neck fracture got 0 because they were too painful to perform any hip joint action and needed to stay in bed. The patients in the ultrasound group got $22.9 \pm 14.7$, and the control group was $24.2 \pm 15.1$ ( $P$ $=0.640 \otimes 0.05, t=0.468$ ), all patients had poor hip joint function preoperatively (the Harris score $\ 70$ ). At 1month and 3 months, the hip Harris scores were $79.6 \pm 3.5$ (72 85) and $89.6 \pm 3.4(82 \sim 95)$ for the ultrasound group and, $80.0 \pm 3.7(72 \sim 86)$ and $90.0 \pm 3.8(84 \sim 96)$ for the control group, respectively. No significant difference was detected between the two groups. The time of operation was also no significant difference between these two groups, which indicated that the ultrasound identification of the LFCN was accomplished before operation and did not dramastically impact the surgical procedure and 
treatment effect. Also, no participant suffered from infection, poor wound healing, prosthetic loosening, or fracture around the prosthesis.

At 1 month after the operation, 2 (3.6\%) patients reported numbness or dull sensation in the cutaneous area of the anterolateral thigh in the ultrasound group, and the symptoms area was $33.0 \pm 10.8(21.0$ 42.0) $\mathrm{cm}^{2}$, and the region was located in the lateral area of the incision. The LFCN of the two patients had three branches at the end, and the lateral branch passed the TFL. In the control group, 15 (24.2\%) patients described abnormal symptoms in the anterolateral thigh, including numbness (15), dull sensation (15), and tingling or pain (4) in the 1st month follow-up, which was significantly higher than that in the ultrasound group. The area of abnormal symptoms of the control group was $133.1 \pm 104.9(49 \sim 375)$ $\mathrm{cm}^{2}$, which was significant larger than that of ultrasound guiding group. At 3 months, both the number of patients and the area of abnormal symptoms did not show any obvious change in the ultrasound group. In the control group, the tingling or pain disappeared completely at 3 months; in 2/4 patients suffering from tingling or pain, the normal sensation was restored. Also the area of numbness or dull sensation did not show a significant difference as compared to that at 1 month after the operation, while the area of abnormal symptoms of the control group was $132.7 \pm 112.9(49 \sim 375.0) \mathrm{cm}^{2}$. (Table 3)

Table 3

Rate of LFCN injury, sensory disturbance area, and Harris scores at 1 and 3 months after operation in the ultrasound and control groups.

\begin{tabular}{|c|c|c|c|c|c|c|c|c|c|}
\hline & \multicolumn{3}{|c|}{ Ultrasound group } & \multicolumn{3}{|c|}{ Control group } & \multirow{2}{*}{$\begin{array}{l}P_{\text {preop }} \\
t_{\text {preop }}\end{array}$} & \multirow{2}{*}{$\begin{array}{l}P_{1} \\
t_{1} / \chi^{2}{ }_{1}\end{array}$} & \multirow{2}{*}{$\begin{array}{l}P_{3} \\
t_{3} / \\
x_{3}^{2}\end{array}$} \\
\hline & Preop & $\begin{array}{l}1 \\
\text { month }\end{array}$ & $\begin{array}{l}3 \\
\text { months }\end{array}$ & Preop & $\begin{array}{l}1 \\
\text { month }\end{array}$ & $\begin{array}{l}3 \\
\text { months }\end{array}$ & & & \\
\hline \multirow[t]{2}{*}{$\begin{array}{l}\text { LFCN } \\
\text { injury }\end{array}$} & \multirow[t]{2}{*}{0} & \multirow[t]{2}{*}{$\begin{array}{l}2 \\
(3.4 \%)\end{array}$} & \multirow[t]{2}{*}{$\begin{array}{l}2 \\
(3.4 \%)\end{array}$} & \multirow[t]{2}{*}{0} & \multirow[t]{2}{*}{$\begin{array}{l}15 \\
(25.9 \%)\end{array}$} & \multirow[t]{2}{*}{$\begin{array}{l}13 \\
(22.4 \%)\end{array}$} & \multirow[t]{2}{*}{-} & 0.001 & 0.005 \\
\hline & & & & & & & & $\begin{array}{l}\chi_{1}^{2}= \\
11.893\end{array}$ & $\begin{array}{l}\chi^{2}{ }_{3}= \\
9.471\end{array}$ \\
\hline \multirow{2}{*}{$\begin{array}{l}\text { Area } \\
\left(\mathrm{cm}^{2}\right)\end{array}$} & \multirow[t]{2}{*}{0} & \multirow{2}{*}{$\begin{array}{l}39.0 \pm \\
4.2 \\
(36.0 \\
\sim \\
42.0)\end{array}$} & \multirow{2}{*}{$\begin{array}{l}39.0 \pm \\
4.2 \\
(36.0 \sim \\
42.0)\end{array}$} & \multirow[t]{2}{*}{0} & \multirow{2}{*}{$\begin{array}{l}133.1 \pm \\
104.9 \\
(49.0 \sim \\
375.0)\end{array}$} & \multirow{2}{*}{$\begin{array}{l}132.7 \pm \\
112.9 \\
(49 \sim \\
375.0)\end{array}$} & \multirow[t]{2}{*}{-} & $\begin{array}{l}<.001 \\
0.001\end{array}$ & \multirow{2}{*}{$\begin{array}{l}0.002 \\
t_{3}= \\
4.139\end{array}$} \\
\hline & & & & & & & & $\begin{array}{l}t_{1}= \\
4.048\end{array}$ & \\
\hline \multirow{2}{*}{$\begin{array}{l}\text { Harris } \\
\text { score }\end{array}$} & \multirow{2}{*}{$\begin{array}{l}22.9 \\
\pm 14.7 \\
(0 \sim \\
48)\end{array}$} & \multirow{2}{*}{$\begin{array}{l}79.6 \pm \\
3.5 \\
(72 \sim \\
85)\end{array}$} & \multirow{2}{*}{$\begin{array}{l}89.6 \pm \\
3.4(82 \\
\sim 95)\end{array}$} & \multirow{2}{*}{$\begin{array}{l}24.2 \\
\pm 15.1 \\
(0 \sim \\
51)\end{array}$} & \multirow{2}{*}{$\begin{array}{l}80.0 \pm \\
3.7(72 \\
\sim 86)\end{array}$} & \multirow{2}{*}{$\begin{array}{l}90.0 \pm \\
3.8(84 \\
\sim 96)\end{array}$} & 0.640 & 0.517 & 0.518 \\
\hline & & & & & & & 0.468 & $\begin{array}{l}t_{1}= \\
0.651\end{array}$ & $\begin{array}{l}t_{3}= \\
0.649\end{array}$ \\
\hline \multirow{2}{*}{$\begin{array}{l}\text { Time of } \\
\text { operation }\end{array}$} & \multirow{2}{*}{\multicolumn{2}{|c|}{$84.8 \pm 5.5$}} & & \multirow{2}{*}{\multicolumn{2}{|c|}{$82.2 \pm 5.2$}} & & \multicolumn{2}{|l|}{0.638} & \\
\hline & & & & & & & $t=2.67$ & & \\
\hline
\end{tabular}

LFCN, the Lateral Femoral Cutaneous Nerve. 


\section{Discussion}

As a mini-invasion approach, DAA for THA is increasingly popular in the clinic because of soft tissue preservation using the inter-muscular and nervous plane,allowing for more reproducible and precise cup placement in supine posion, and fast functional discovery after surgery[9, 22]. However, the abnormal sensation caused by LFCN injury on the area of anterolateral thigh, including numbness, paresthesia, and pain, is the most common complaint of patients after surgery[14,23]. The main reasons that result in

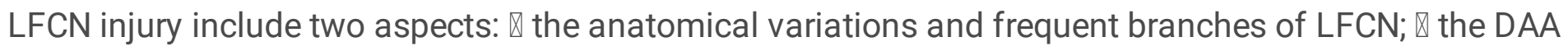
approach close to the path of LFCN. In the proximal femur, LFCN courses the intermuscular space between the TFL and sartorius muscles, which is also the surgical field [17, 24, 25]. These two factors increase the risk of cutting or suturing of LFCN interoperation, which are the main reasons for LFCN injury.

Ultrasound is the first choice level technique to image the nerve according to the last Gruidelines of the European Society of Musculoskeletal Radiology (ESSR) [26] and widely applicated in ultrasound-guided injections around the nerve $[27,28]$, we attempted to use ultrasound to identify the 3D distribution, which would help to avoid damaging LFCN from cutting to the suture. This is different from traditional skin marking protocol using ultrasound guidance. Skin marking follows the skin movement especially in elderly or patients with loose skin, which causes uncertain localization of LFCN on the skin surface. The $3 \mathrm{D}$ distribution identification provides three types of data to the surgeons, including path, depth, and distance to TFL in the surgical region. Subsequently, the $D$ was $\leq 2 \mathrm{~cm}$ from the point of LFCN exit (between the medial of ASIS and lateral 1/3rd of the inguinal ligament) to the ASIS. The depth of LFCN to the skin was deeper in the far end than that in the exit location, and this phenomenon was obvious in the obese patients who had thick subcutaneous fat. In the surgical region, LFCN was a single branch in the exit point, and always in the fascia latae with a bilayer structure. In the distal end of ASIS within $10 \mathrm{~cm}$, LFCN mainly had a single branch, followed by two types of branches and then, three or more branch types. The more branches the LFCN had, the closer to TFL was, and the higher risk of LFCN injury. Three or more branches of LFCN were collectively termed as fan-type. In this study, two patients were identified fan-type, and the lateral branch of LFCN of 3 patients was cut as it obstructed the operation exposure. Another study had been reported that LFCN named as the fan-type injury cannot be avoided in DAA surgical dissections[18]. Herein, it was also demonstrated that the surgeon had to cut some branches of LFCN to acquire adequate surgical vision field though found them in patients with fan-type LFCN. The average distance to medical TFL was about $15 \mathrm{~mm}$, and it became closer to TFL from proximal to distal. If there is more than one branch, the lateral branch of LFCN passes the lateral side of TFL, indicating that the incision of DAA should be located at least $10 \mathrm{~mm}$ distance from the medical side of TFL. The skin was marked with the line of the medical side of TFL was the line of ASIS and lateral condyle of the femur. In addition, to avoid the suturing injury of LFCN and result in meralgia paresthetica, another $5 \mathrm{~mm}$ distance from LFCN to incision should be added for the suture fascia layer. Therefore, the DAA incision needs to be localized $15 \mathrm{~mm}$ to the lateral side of the line of ASIS and lateral condyle of the femur. 
The 3D location of LFCN using non-invasiveness of ultrasonography, which was not just a traditionally projection onto the skin[29], provided multidimensional distribution information for surgeons, with respect to the LFCN during incision of the skin and opening and closing of the fascia layer. Compared with no 3D location, the identification of LFCN was a great option to significantly decrease the rate of DAA-induced LFCN injury. Here, $10 \mathrm{MHz}$ transducer was used to detect the stem of LFCN firstly, and then higher frequencies probe was used to avoid omiting some superficial branches because that different frequencies provided different sencitivities in various depths [30]. Similar studies, which focused on the treatment of meralgia paresthetica of LFCN or nerve block conduction using ultrasound guiding technique, also demonstrated that ultrasonographic images provided precious and visible position that would be helpful to a safe and effective treatment, especially for nerves with anatomical variation and some branches [31-33].

In addition, almost the patients who suffered from LFCN injury in the control group complained of troubled paresthesia in first follow-up. After patients were explained and made comfortable, they could understand and accept the situation. Therefore, the identification of LFCN before surgery was also an optimal option for patients, especially for those with the fan-type LFCN. Based on the location of DAA incision and LFCN distribution, the surgeons could easily evaluate the risk level of LFCN injury during the operation, and explain to the patients that the branches of LFCN will be injured and some symptoms of sensory disturbance will be observed after the operation. Thus, the patients will accept the situation and let their mind at ease, which would alleviate the conflicts between doctors and patients.

Nevertheless, the method of identification of LFCN using ultrasound also has some limitations. It needs an experienced ultrasonography doctor to search for LFCN due to its variation. The ultrasound identification examination also adds to the cost of inpatient. Also, additional time and energy would be needed to record the map of LFCN on the skin and some parameters due to individual variation. In this study, we identified the location of LFCN preoperatively but did not identify whether it was intact or injured postoperatively. The chief complaint of the patients was the only evidence to evaluate the situation of LFCN in the follow-up. Therefore, the rate of injury in LFCN may be influenced by the subjective factor of patients. In addition, as for age or BMI, there was no significant difference between these two groups, but age or BMI were possible risk factors of damaging the LFCN because young patients with strong muscles and obsese patient need strong force to pull their incisions to obtain good surgical vision. And pulling during operation was a risk to increase the rate of nerve injury.

\section{Conclusions}

Before surgery, it is a choice for the surgeons for DAA-THA to identify the 3D distribution of LFCN using ultrasound. Based on the marking on the skin and location to TFL, surgeons can make a preoperation plane and select an incision to reduce the rate of LFCN injury, or even inform the patients before surgery that some branches of LFCN will be cut during the operation. This would alleviate their anxiety and the conflicts between the doctors and patients postoperatively. In addition, the anatomical parameters from 
the ultrasound group also provided a general reference for doctors who could not implement ultrasoundguided identification of LFCN for every patient who underwent DAA-THA.

\section{Abbreviations}

LFCN: Lateral Femoral Cutaneous Nerve; DAA: Direct Anterior Approach; THA: Total Hip Arthroplasty; 3D: Three-dimensional; TFL: Tensor Fasciae Latae; ASIS: Anterior Superior Iliac Spine; MRI: Magnetic Response Image; IL: Inguinal Ligament; FNF: Femoral Neck Fracture; FHN: Femoral Head Necrosis; OA: Osteoarthritis; BMI: Body Mass Index.

\section{Declarations}

\section{Ethics approval and consent to participate}

The study was approved by the Ethical Committee of Nanjing Drum Tower Hospital. Writing informed consents were obtained from all patients who accepted this surgical procedure before operation.

\section{Consent to publish}

Not applicable.

\section{Availability of data and materials}

The datasets used and/or analysed during the current study are available from the corresponding author on reasonable request.

\section{Competing interests}

The authors declare that they have no competing interests.

\section{Funding}

This work was supported by the National Science Foundation of China (81972124), Medical Technology and Development Key Foundation of Nanjing (ZKS18020).

\section{Authors' contributions}

YZ and YY designed the study, identified the distribution of LFCN using ultrasound and wrote the paper; YXW and YS collected and analyzed the data; ZKZ collected and analyzed the data; QJ supervised the 
study and corrected the manuscript; DYC performed the surgery and supervised the study. All authors read and approved the final manuscript

\section{Acknowledgements}

Not applicable.

\section{References}

1. Wu H, Cheng WD, Jing J. Total hip arthroplasty by direct anterior approach in the lateral position for the treatment of ankylosed hips. Eur J Orthop Surg Traumatol. 2020;30(6):993-1001.

2. Chen W, Sun JN, Zhang Y, Zhang Y, Chen XY, Feng S. Direct anterior versus posterolateral approaches for clinical outcomes after total hip arthroplasty: a systematic review and meta-analysis. J Orthop Surg Res. 2020;15(1):231.

3. Trivellin G, Assaker A, Vacchiano A, Cominelli D, Meyer A. Direct anterior total hip arthroplasty: a retrospective study, Acta bio-medica. Atenei Parmensis. 2020;91:98-102.

4. Vasantharao P, Fenbury D, Khan R, Fick D, Dalgleish S, Finsterwald M, Castle H, Haebich S. Anterior approach to hip replacement and associated complications: an independent review, HIP International (2020) 112070002094845.

5. Cichos K, Mabry S, Spitler C, McGwin G, Quade J, Ghanem E. A Comparison Between the Direct Anterior and Posterior Approaches for Total Hip Arthroplasty Performed for Femoral Neck Fracture, Journal of orthopaedic trauma (2020).

6. Herndon CL, Drummond N, Sarpong NO, Cooper HJ, Shah RP, Geller JA. Direct anterior versus minianterolateral approach for primary total hip arthroplasty: early postoperative outcomes and complications. Arthroplast Today. 2020;6(2):257-61.

7. Kawano T, Kijima H, Yamada S, Konishi N, Kubota H, Tazawa H, Tani T, Suzuki N, Kamo K, Okudera Y, Fujii M, Sasaki K, Iwamoto Y, Nagahata I, Miura T, Miyakoshi N, Shimada Y. A Comparison of the Incidences of Venous Thromboembolism after Total Hip Arthroplasty between the Direct Anterior Approach and the Direct Lateral Approach, Especially in the Early Period after Introduction of the Direct Anterior Approach. Adv Orthop. 2020;2020:4649207.

8. Siljander MP, Whaley JD, Koueiter DM, Alsaleh M, Karadsheh MS. Length of Stay, Discharge Disposition, and 90-Day Complications and Revisions Following Primary Total Hip Arthroplasty: A Comparison of the Direct Anterior, Posterolateral, and Direct Superior Approaches. The Journal of Arthroplasty. 2020;35(6):1658-61.

9. Wilson JM, Schwartz AM, Farley KX, Anastasio AT, Bradbury TL, Guild GN 3rd, Postoperative acetabular component position in revision hip arthroplasty: a comparison of the anterior and posterior approaches, Hip Int (2020) 1120700020942451. 
10. Dall'Oca C, Ceccato A, Cresceri M, Scaglia M, Guglielmini M, Pelizzari G, Valentini R, Magnan B. Facing complications of direct anterior approach in total hip arthroplasty during the learning curve, Acta bio-medica. Atenei Parmensis. 2020;91:103-9.

11. Goulding K, Beaule PE, Kim PR, Fazekas A. Incidence of lateral femoral cutaneous nerve neuropraxia after anterior approach hip arthroplasty. Clin Orthop Relat Res. 2010;468(9):2397-404.

12. Vajapey SP, Morris J, Lynch D, Spitzer A, Li M, Glassman AH. Nerve Injuries with the Direct Anterior Approach to Total Hip Arthroplasty. JBJS Reviews. 2020;8(2):e0109.

13. Bhargava T, Goytia R, Jones L, Hungerford M. Lateral femoral cutaneous nerve impairment after direct anterior approach for total hip arthroplasty. Orthopedics. 2010;33(7):472.

14. Macheras GA, Christofilopoulos P, Lepetsos P, Leonidou AO, Anastasopoulos PP, Galanakos SP. Nerve Injuries in Total HIP Arthroplasty with a Mini Invasive Anterior Approach. HIP International. 2016;26(4):338-43.

15. Ozaki Y, Homma Y, Sano K, Baba T, Ochi H, Desroches A, Matsumoto M, Yuasa T, Kaneko K. Small femoral offset is a risk factor for lateral femoral cutaneous nerve injury during total hip arthroplasty using a direct anterior approach. Orthop Traumatol Surg Res. 2016;102(8):1043-7.

16. Palamar D, Terlemez R, Akgun K. Ultrasound-Guided Diagnosis and Injection of the Lateral Femoral Cutaneous Nerve with an Anatomical Variation, Pain practice: the. official journal of World Institute of Pain. 2017;17(8):1105-8.

17. Rudin D, Manestar M, Ullrich O, Erhardt J, Grob K. The Anatomical Course of the Lateral Femoral Cutaneous Nerve with Special Attention to the Anterior Approach to the Hip Joint, The Journal of bone and joint surgery. American volume. 2016;98(7):561-7.

18. Ozaki Y, Baba T, Homma Y, Tanabe H, Ochi H, Bannno S, Watari T, Kaneko K. Preoperative ultrasound to identify distribution of the lateral femoral cutaneous nerve in total hip arthroplasty using the direct anterior approach. SICOT J. 2018;4:42.

19. Palamar D, Terlemez R, Akgun K. Ultrasound-Guided Diagnosis and Injection of the Lateral Femoral Cutaneous Nerve with an Anatomical Variation. Pain Pract. 2017;17(8):1105-8.

20. Park BJ, Joeng ES, Choi JK, Kang S, Yoon JS, Yang SN. Ultrasound-guided lateral femoral cutaneous nerve conduction study. Ann Rehabil Med. 2015;39(1):47-51.

21. Onat S, Ata A, Ozcakar L. Ultrasound-Guided Diagnosis and Treatment of Meralgia Paresthetica. Pain Physician. 2016;19(4):E667-9.

22. Cao J, Zhou Y, Xin W, Zhu J, Chen Y, Wang B, Qian Q. Natural outcome of hemoglobin and functional recovery after the direct anterior versus the posterolateral approach for total hip arthroplasty: a randomized study. J Orthop Surg Res. 2020;15(1):200.

23. Weynandt C, Kowski A, Perka C, Rakow A, [Nerve Injuries in Hip and Knee Arthroplasty - Risk Factors, Diagnostic and Therapeutic Approaches], Zeitschrift fur Orthopadie und Unfallchirurgie (2020).

24. Peng L, Zeng Y, Wu Y, Zeng J, Liu Y, Shen B. Clinical, functional and radiographic outcomes of primary total hip arthroplasty between direct anterior approach and posterior approach: a systematic review and meta-analysis. BMC Musculoskelet Disord. 2020;21(1):338. 
25. Liu Zy, Zhang J, Wu St, Li Zq, Xu Zh, Zhang X, Zhou Y, Zhang Y. Direct Anterior Approach in Crowe Type. III-IV.

26. Developmental Dysplasia of the Hip: Surgical Technique and 2 years Follow-up from Southwest China, Orthopaedic Surgery. (2020). Sconfienza LM, Albano D, Allen G, Bazzocchi A, Bignotti B, Chianca V, Facal de Castro F, Drakonaki EE, Gallardo E, Gielen J, Klauser AS, Martinoli C, Mauri G, McNally E, Messina C, Miron Mombiela R, Orlandi D, Plagou A, Posadzy M, de la Puente R, Reijnierse M, Rossi F, Rutkauskas S, Snoj Z, Vucetic J, Wilson D, Tagliafico AS. Clinical indications for musculoskeletal ultrasound updated in 2017 by European Society of Musculoskeletal Radiology (ESSR) consensus. Eur Radiol. 2018;28(12):5338-51.

27. Klauser A, Abd Ellah M, Halpern E, Sporer I, Martinoli C, Tagliafico A, Sojer M, Taljanovic M, Jaschke W. Meralgia paraesthetica: Ultrasound-guided injection at multiple levels with 12-month follow-up. European radiology. 2016;26(3):764-70.

28. Dettori N, Choudur H, Chhabra A. Ultrasound-Guided Treatment of Peripheral Nerve Pathology. Semin Musculoskelet Radiol. 2018;22(3):364-74.

29. Ellis J, Schneider JR, Cloney M, Winfree CJ. Lateral Femoral Cutaneous Nerve Decompression Guided by Preoperative Ultrasound Mapping. Cureus. 2018;10(11):e3652.

30. Albano D, Aringhieri G, Messina C, De Flaviis L, Sconfienza LM. High-Frequency and Ultra-High Frequency Ultrasound: Musculoskeletal Imaging up to $70 \mathrm{MHz}$. Semin Musculoskelet Radiol. 2020;24(2):125-34.

31. Ahmed A, Arora D, Kochhar AK. Ultrasound-guided alcohol neurolysis of lateral femoral cutaneous nerve for intractable meralgia paresthetica: a case series. Br J Pain. 2016;10(4):232-7.

32. Kloosterziel ME, Tavy DLJ, Arends S, Zijdewind JM, Zwet EW, Wirtz PW. Meralgia paresthetica: Nerve stimulator-guided injection with methylprednisolone/lidocaine, a double-blind randomized placebocontrolled study. Muscle Nerve. 2020;61(6):788-91.

33. Nielsen TD, Moriggl B, Barckman J, Kolsen-Petersen JA, Soballe K, Borglum J, Bendtsen TF. The Lateral Femoral Cutaneous Nerve: Description of the Sensory Territory and a Novel UltrasoundGuided Nerve Block Technique. Reg Anesth Pain Med. 2018;43(4):357-66.

\section{Figures}



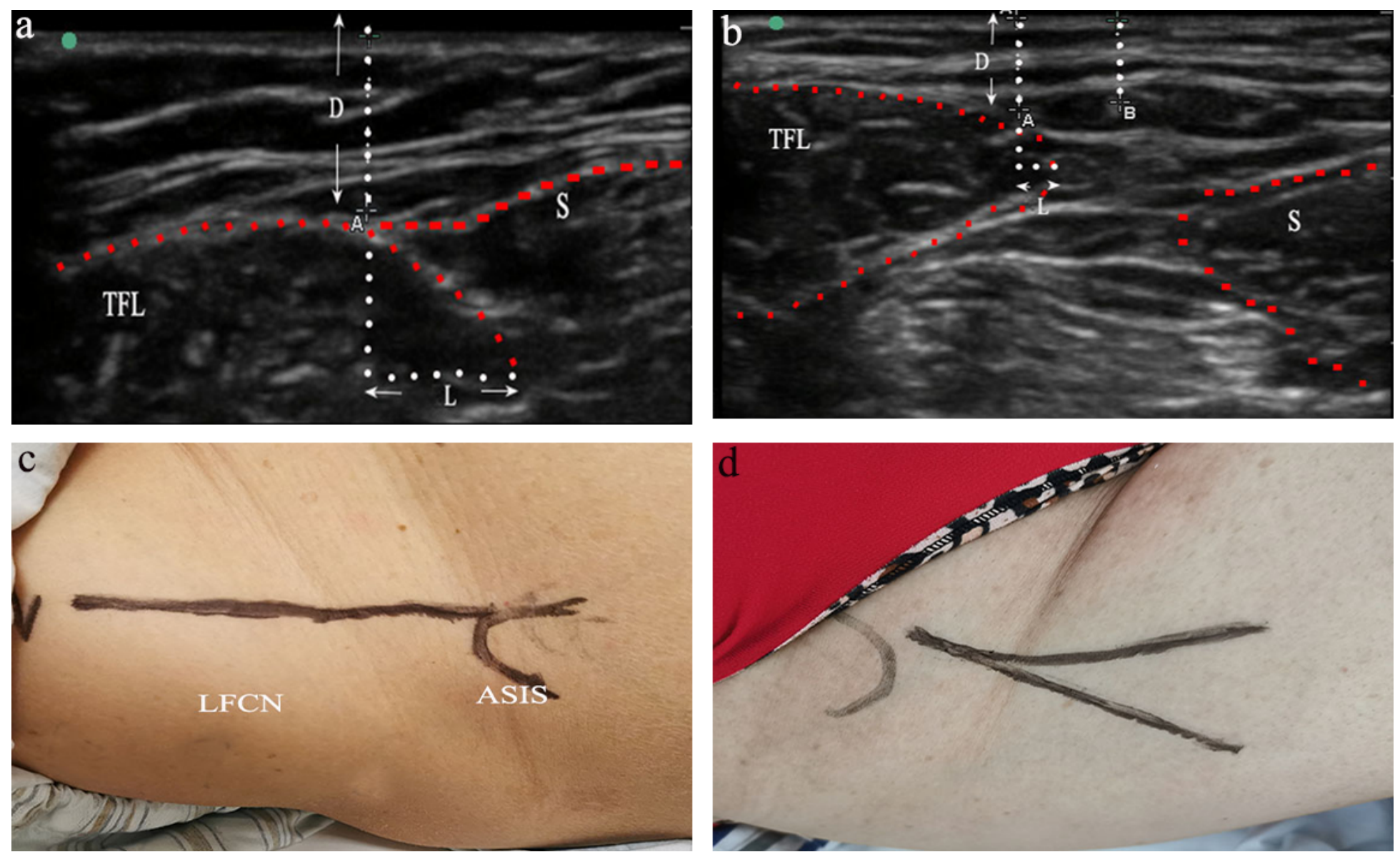

\section{Figure 1}

3D identification of LFCN with Doppler ultrasound photograph. a, One stem nerve ultrasound image of LFCN. b. Two branches of the nerve ultrasound image of LFCN. A and B were cross sections of the branches of LFCN. $L$ was the length from LFCN to the medical side of TFL, and D was the distance to the skin surface. c, d. The maps of one branch type and Fan type of LFCN on the skin surface.
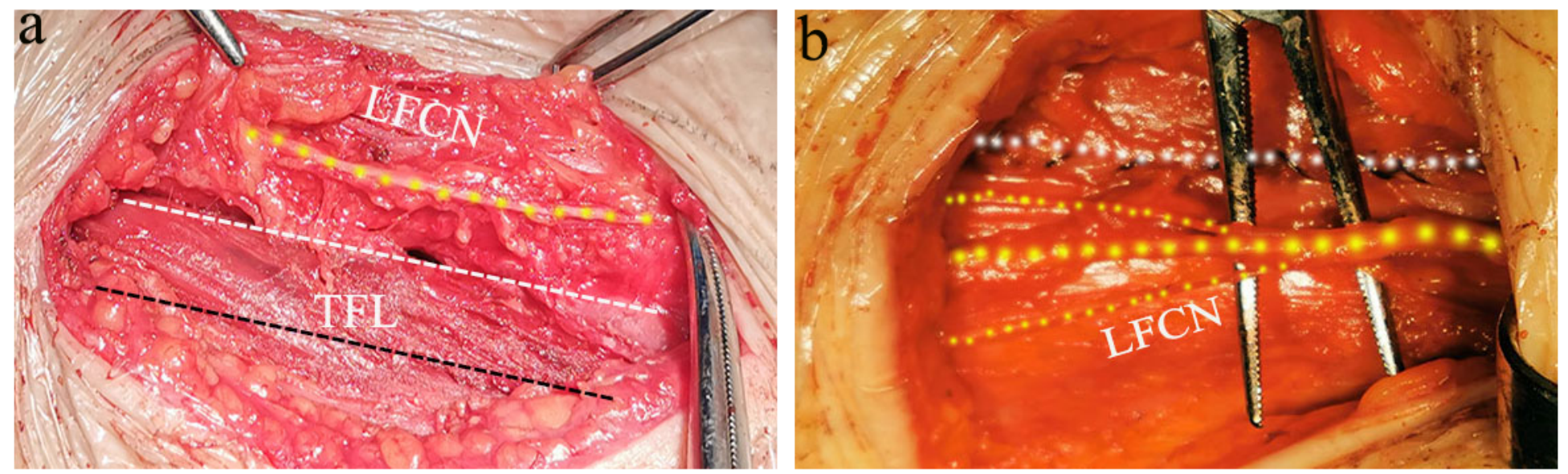

\section{Figure 2}

The intraoperative anatomy of LFCN showed its relative location relationships between incision and TFL. $a$, The single branch type of LFCN; b, The Fan type of LFCN. Yellow and white dots indicated LFCN path 
and sutured incision, white broken line and black line showed the medical border of TFL and incision of muscular fasciae
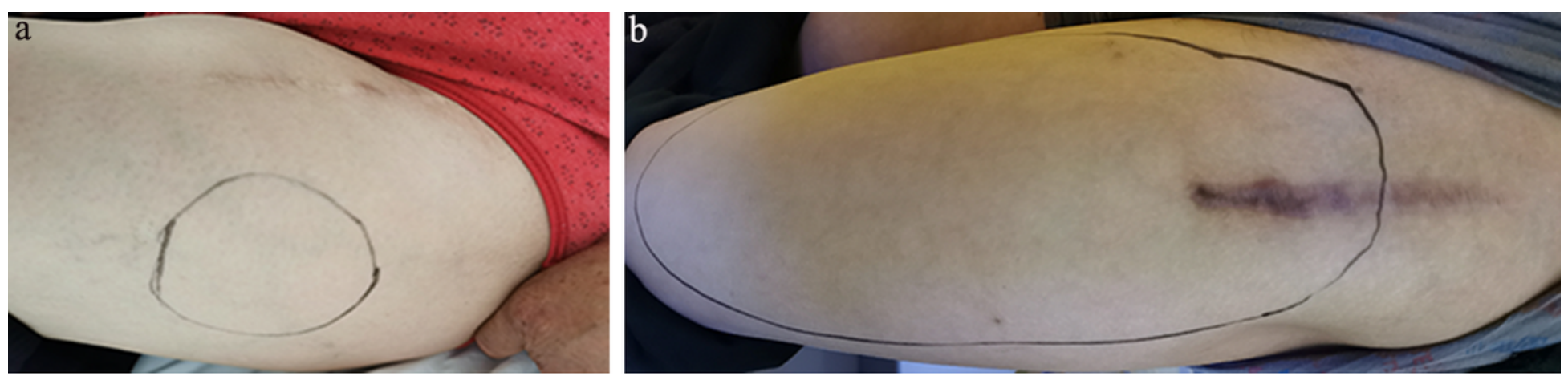

\section{Figure 3}

Sensory abnormal area after surgery. a, The abnormal sensation region on the lateral side of incision in the ultrasound group. b, The abnormal sensation region was located on the region of LFCN in the control group. 